\title{
A Novel Actuated Tether Design for Rescue Robots Using Hydraulic Transients
}

\author{
Douglas P. Perrin \\ Division of Engineering and \\ Applied Sciences \\ Harvard University \\ Pierce Hall, 29 Oxford Street \\ Cambridge, Massachusetts 02138 \\ Email: dperrin@deas.harvard.edu
}

\author{
Albert Kwon \\ Milton Academy \\ Milton, Massachusetts
}

\author{
Robert D. Howe \\ Division of Engineering and \\ Applied Sciences \\ Harvard University \\ Pierce Hall, 29 Oxford Street \\ Cambridge, Massachusetts 02138 \\ Email: howe@deas.harvard.edu
}

\begin{abstract}
In the world of search and rescue robotics, particularly for search, smaller is better. Small robots can get in to tighter places and are more maneuverable. With diminishing size, however, providing adequate power and communications becomes a problem. Communication is problematic if the disaster site is a collapsed building were transmitted signals have to travel through layers of concrete and steel. Tethers are good for providing power and communication, but tethers get stuck and small robots have difficulty with the added drag of the tether. This work proposes a self actuating tether capable of moving its own weight and remaining free while traversing around corners. The tether motion is due to induced high pressure water transients formed by rapidly arresting flow through a hose. A number of tests performed on a constructed tether prototype are presented, a simplified model of the water transients to better understand design parameters is outlined and simulated, and force measurements are collected to validate the simulation results.
\end{abstract}

\section{INTRODUCTION}

A long standing goal of mobile robotics has been to allow robots to work in environments unreachable or too hazardous to risk human lives. Urban search and rescue is one of the most hazardous environments imaginable; victims are often in unreachable locations buried beneath rubble. Rescue robotics is the application of robotics to the search and rescue domain. The goal of rescue robotics is to extend the capabilities of human rescuers while also increasing their safety. Rescue robots were put to the test at the World Trade Center (WTC) disaster, on Sept. 11, 2001. In the chaotic environment of the collapsed towers, radio controlled and tethered robots were deployed eight times by the Center For Robotic Assisted Search and Rescue (CRASAR). These robots varied from shoe box sized to suitcase dimensions. During the course of their deployment the robots encountered a number of difficulties involving radio transmission inefficiency [1] [2], poor maneuverability [3], and tether management [1]. In this work, we will explore the properties of hydraulic transients (also known as water hammer) as a method of tether actuation. By actuating the tether we hope to overcome some the aforementioned difficulties of using tether robots in unstructured environments.

When a communication failure caused temporary loss of an untethered teleoperated robot at the WTC, this highlighted the problem with using radio frequencies at disaster sites. Usable frequencies are limited since most are reserved by emergency response agencies. Additionally, the thick wall of debris often obstructed radio communication between the operator and the robot [2]. These issues made point-to-point navigation of the smaller tethered robots more reliable and useful than that of the untethered robots [3].

Lack of mobility also hindered the robotic search and rescue effort. Track-drives also limited robots mobility once inserted in the rubble [3]. Tethers improve mobility since robots can be safely lowered or raised vertically where the untethered robots can not [1] [3]. Similarly, the tethers can be used for emergency robot recovery as was demonstrated at the WTC when malfunctioning robots were recovered by pulling them out by their tethers. The negative side of using tethers is increased drag and a tendency to catch on obstacles. Tethers limit the depth and path of smaller robots. Since the robots were not able to enter ports smaller than their size, the larger robots were less useful than their smaller counterparts. Tethers also require more caution on the part of the operator to avoid breaking or locking the tether on debris [3]. The use of tethers at the WTC also put operators at risk since tether managers often had to work close to structures with questionable stability during the deployment [1].

What is needed is a tether design that actively prevents or solves entanglement problems thus providing more mobility and range for the rescue robots at a reasonable price. The proposed water actuated tether achieves these goals. By inducing movement along the tether it can free itself if caught and reduce the overall likelihood of becoming caught in the first place. This motion is created by arresting the flow of water in the tether. A simple block diagram of the tether design in shown in Fig. 1. By rapidly closing a valve momentum is transfered from flowing water to the valve and surrounding hose. The resulting force spike creates a jerking motion in the tether. If biased in one direction this jerking motion would not only keep the tether free but would provide propulsion as well. With this tether design smaller robots will be able to penetrate further in to a disaster site while leaving the operator at a much safer distance. 


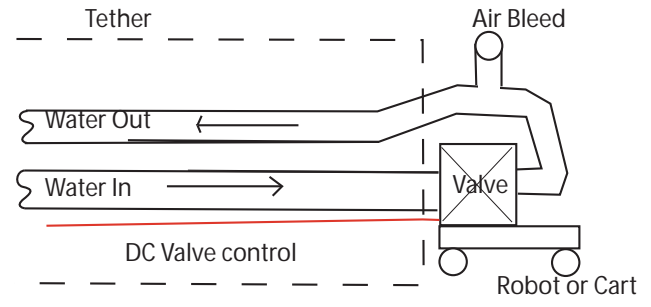

Fig. 1. Block diagram of tether design

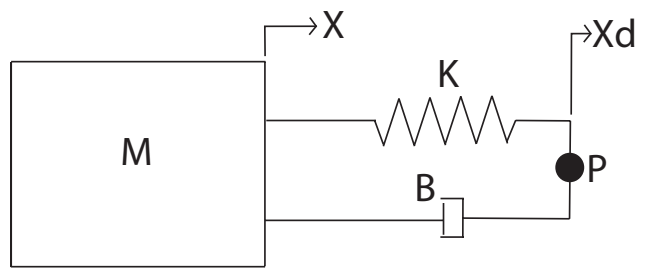

Fig. 2. Lump mass model of water hammer

\section{Hydraulic TRAnsients (WATER Hammer)}

Water hammer is a phenomenon that occurs in pipelines when flow is cut off suddenly. A near instantaneous closure of a valve or a faucet creates a shock wave that travels through the water line causing metal pipes and joints to expand and in the worst case rupture. Repetition may damage pipes, valves, and pipe joints. In order to minimize damage, air-filled chambers called "air cushions" are added to household plumbing so that the shock waves dissipate. An everyday example of these transients may be observed when fueling an automobile. When the valve handle at the nozzle is released, the fuel hose jerks due to the sudden cessation of the flow. It is this jerk that is harnessed for actuation of our tether.

Pressure transients are a complex and well-studied fluid mechanical phenomenon; for an introduction see [4]. An indepth discussion is beyond the scope of this paper, and a complete model of water hammer is unnecessary for the design of the actuated tether. In this section a simple model is presented that captures the salient parameter relationship in the water hammer effect.

\section{A. Simplified Model of Water Hammer}

Figure 2 shows the simplified lumped-element water hammer model. This model captures the dynamics due to the rapid cessation of flow, and relates system parameters to the force transient applied to the valve. In water hammer, a volume of liquid is traveling along the hose with initial velocity $\dot{x_{o}}$ when the valve is suddenly closed. A fraction of the water's change in momentum produces a force at the valve, while some energy is transferred to expansion of the compliant hose near the valve and propagation of pressure waves away from the valve. A mass $M$ with position $x$ represents the effective volume of water initially traveling through the hose. A Stiffness element $K$ and damping element $B$ connected to the mass represent the impedance of the hose and energy loss mechanisms including wave propagation and fluid dissipation. In this model we assume the outflow side of the valve is open to air, so there is no effective mass to the right of point $P$. Closure of the valve is then represented as the rapid deceleration of $P$, described by the function $x_{d}(t)$. The resulting force $F$ at $P$ is the force generated at the valve assembly

$$
F=K\left(x_{d}-x\right)+B\left(\dot{x_{d}}-\dot{x}\right)=M \ddot{x} .
$$

The input to this model is the initial water velocity $\dot{x_{o}}$ and the valve closure function $x_{d}(t)$, which we represent as a decaying exponential with time constant $\tau$

$$
\dot{x_{d}}=\dot{x_{o}} \exp ^{\frac{-t}{\tau}} .
$$

This second-order system can be readily integrated to find $F(t)$ for specified values of $\dot{x_{o}}, M, B$ and $K$. The initial velocity $\dot{x_{o}}$ is readily determined from flow rate calculations or measurements. The mass can be expressed as $M=\rho V=$ $\rho A L$, where $\rho$ is the density of water and $V$ represents the volume of water that acts to produce the force transient; due to bending and losses in the hose, $V$ is less than the total volume contained in the hose. $A$ is the cross sectional area of the hose and $\mathrm{L}$ is the effective length of the water mass. To estimate $V$, we can relate it to the flow rate $\dot{V}=A \dot{x}$, so

$$
\frac{d \dot{V}}{d t}=A \frac{d \dot{x}}{d t} .
$$

The force $F$ is then

$$
F=M \frac{d \dot{x}}{d t}=(A L \rho)\left(\frac{1}{A} \frac{d \dot{V}}{d t}\right)
$$

and the impulse is

$$
\begin{aligned}
\int_{\text {open }}^{\text {closed }} F d t & =L \rho \int \frac{d \dot{V}}{d t} \\
& =L \rho|\dot{V}|_{\text {open }}^{\text {closed }} .
\end{aligned}
$$

The effective length of the water mass is then

$$
L=\frac{\int_{\text {open }}^{\text {closed }} F d t}{\dot{V}_{0} \rho}
$$

where $\dot{V}_{0}$ is initial volume flow rate. Integration of the measured force signal provides an estimate of the effective length of the water mass that acts to produce the force transient.

Figure 3 shows the results of model calculations for values of $M=.0431 \mathrm{~kg}, B=5 \mathrm{Ns} / \mathrm{m}$, and $K=4000 \mathrm{~N} / \mathrm{m}$. These values were chosen to match the observed force signals in the experiments reported below. Separate curves represent three values of the valve closing time constant $\tau$ from 5 to $45 \mathrm{~ms}$, the expected range for commercial valves of the type used in the experiments. The simulation shows that shorter valve closure times result in greater initial force impulses at the valve, with essentially constant oscillation frequency. Figure 4 shows model forces for two hose diameters, assuming constant volume flow rate. Peak forces are similar, but the smaller diameter hose shows a faster decay. 


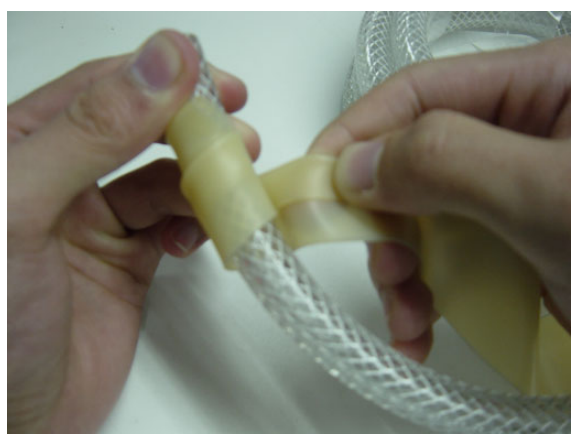

(a)

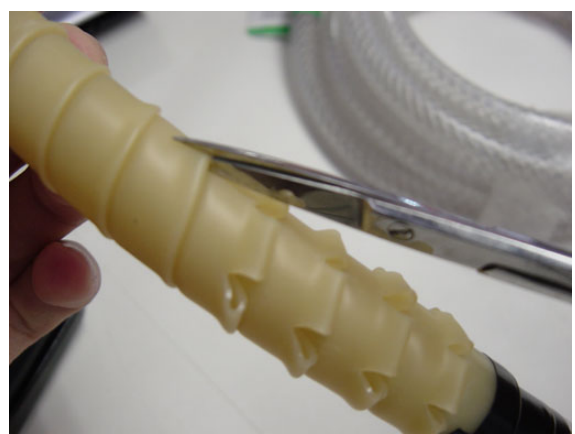

(b)

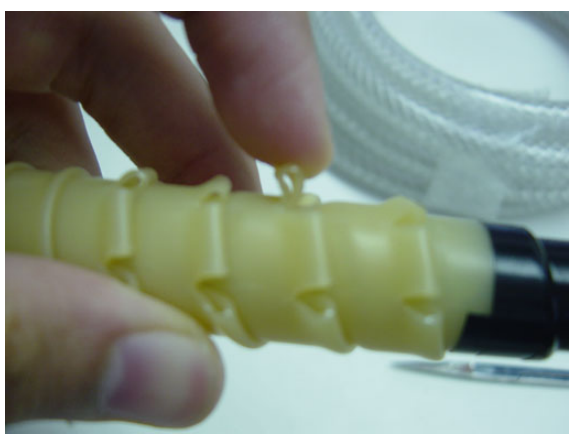

(c)

Fig. 5. Construction steps friction biasing skin.

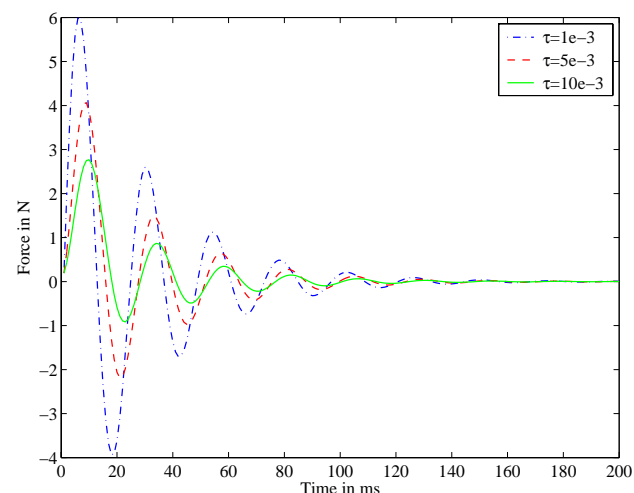

Fig. 3. Simulated forces varying $\tau$ with $M=.0431 \mathrm{~kg}, \dot{x_{0}}=.65 \mathrm{~m} / \mathrm{s}, B=$ $3 \mathrm{Ns} / \mathrm{m}$, and $K=3000 \mathrm{~N} / \mathrm{m}$

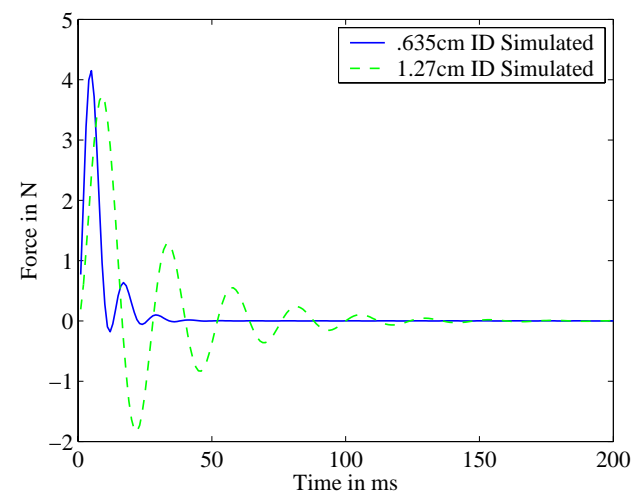

Fig. 4. Simulation of two different hose diameters and a constant flow rate the simulation parameters are: $\tau=1 \times 10^{-3} \mathrm{~s}, B=3 \mathrm{Ns} / \mathrm{m}$, and $K=3000 \mathrm{~N} / \mathrm{m}$. $M=.0431 \mathrm{~kg}$ and $\dot{x_{0}}=.64 \mathrm{~m} / \mathrm{s}$, for the $1.27 \mathrm{~cm}$ ID hose. $M=.0104 \mathrm{~kg}$ and $\dot{x_{0}}=2.567 \mathrm{~m} / \mathrm{s}$ for the $0.635 \mathrm{~cm}$ ID hose

\section{Materials And Methods}

The initial prototype of the tether system consists of a flexible water hose, an electric solenoid valve and a toy vehicle to act as a robot. With this system we conducted three experiments: the first were drag tests using the vehicle to simulate an exploring robot. Second, a directionally biasing skin was tested for efficacy. Last, force measurements were collected to validate the model proposed in section II-A.

\section{A. Robot Drag Tests}

We conducted a number of tests with a 9.6 Volt battery powered toy car as a robot (Figure 8) with the valve (Type 18AR43, Magnatrol ValveCorp., Hawthorn, NJ) mounted directly to the vehicle. The vehicle is capable of pulling with $8 \mathrm{~N}$ of force. These tests showed that the tether can be dragged by a small robot without getting caught on corners. The in-flow and out-flow lines were lashed together to form the tether. The first test was to determine if the tether could unlock itself from a corner or an S curve around obstacles. The second was to see if the addition weight of the water filled tether would make it too heavy for a small robot to drag. The test cases were setup so that the vehicle was unable to move forward on its own accord (Tether turned off). The test was considered a success if the vehicle could move forward with the tether turned on.

\section{B. Skin Design}

A skin was designed to test the hypothesis that having a frictional bias could be used to maximize the directional affects of water hammer oscillation. A skin with a smooth surface in one direction and a rough surface in the opposite direction acts as a ratcheting mechanism allowing the tether to move forward using the jerks from water hammer but not slide back.

A simple frictionally biased skin was constructed using latex rubber. Strips of latex rubber $3 \mathrm{~cm}$ wide were folded and wrapped around the hose. The folded edge was cut to create flaps as shown in Figure 5(a) and 5(b). When the hose slides forward, the flaps stay flat. In the opposite direction, however, the flaps will act against the motion as shown in Fig. 5(c), maximizing the forward motion and minimizing retrograde motion. It should be noted that this design only moves the tether forward and design of a bidirectional skin remains as future work.

\section{Direct Force Measurement}

Force data was collected using a force sensor (Gama model, ATI Inc., Apex, NC) in order to characterize induced forces at the valve. Two different experiments with different diameter hoses were performed. Force data was collected with the valve set up at the same level of the water faucet and with the hose suspended in air (Fig. 6). 


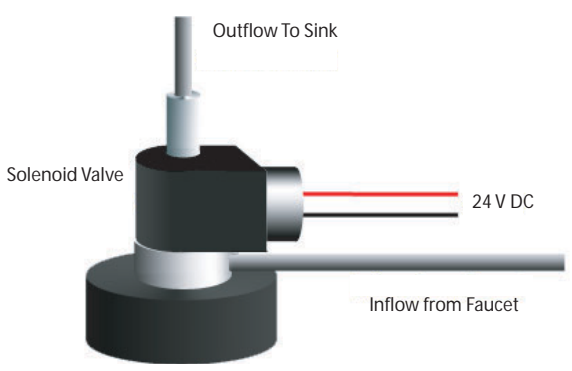

ATI Multi-axis Force/Torque Sensor System

Fig. 6. Configuration of force sensor

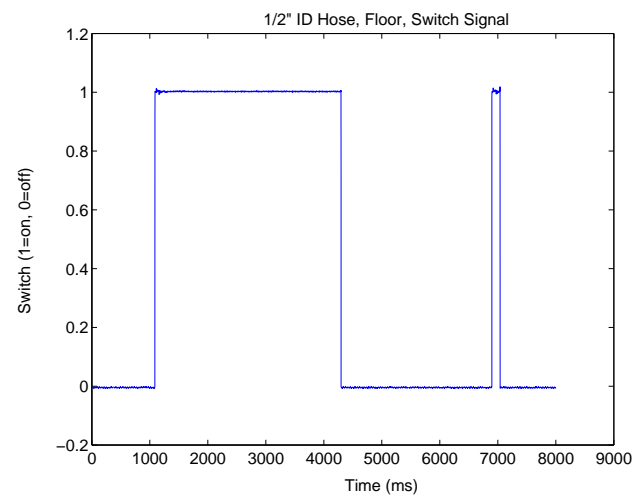

Fig. 7. An example of the valve control signal

For the force measurements the solenoid valve was rigidly mounted to the force sensor and a metal plate was attached to the bottom of the sensor and clamped to an edge of the sink. The out-flow of water was directly dumped into the sink through a $50 \mathrm{~cm}$ long hose. The in-flow hose was approximately $3 \mathrm{~m}$ long. Minimal contact of the hose with any surface was maintained for the experiments.

Data along the force sensors $y$-axis was collected. The $y$ axis was chosen due to the orientation of the valve with respect to the force/torque sensor; the inflow hose was aligned with the sensors $y$-axis and the outflow hose was aligned with the $z$-axis. The test cycle consisted of repeated on and off signals (Fig. 7).

For the first force measurement, a $0.635 \mathrm{~cm}$ internal diameter (ID), $1.14 \mathrm{~cm}$ outer diameter (OD), nylon reinforced vinyl hose was used (cross sectional area of $3.14 \times 10^{-5} \mathrm{~m}^{2}$ ). The valve was positioned near the sink and the hose was suspended. The valve was shut at approximately $650 \mathrm{~ms}$, opened at approximately $1850 \mathrm{~ms}$, and pulsed at $3150 \mathrm{~ms}$.

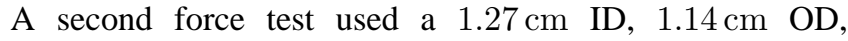
nylon reinforced vinyl hose was used (cross sectional area of $1.3 \times 10^{-4} \mathrm{~m}^{2}$ ). The valve was shut at approximately $625 \mathrm{~ms}$, opened at approximately $1300 \mathrm{~ms}$, and pulsed at approximately $1850 \mathrm{~ms}$.

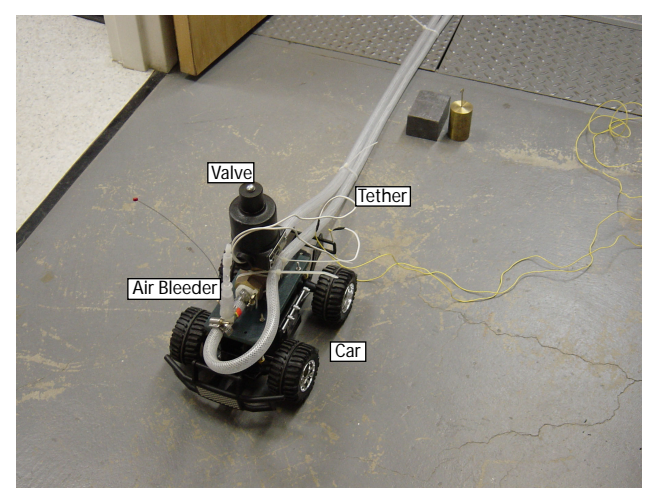

Fig. 8. Vehicle with a valve and tether attached

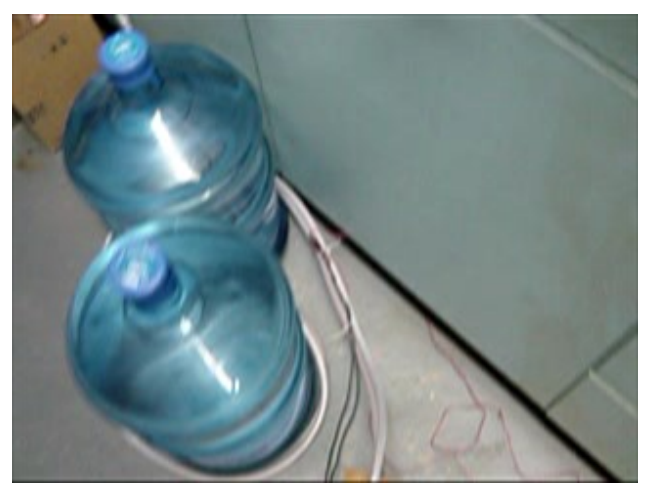

Fig. 9. Tether wrapped around cylinders

\section{EXPERIMENTAL RESULTS}

\section{A. Robot Drag Tests}

Every turn made by a rescue robot in a maze of concrete, metal, and dust debris increases the possibility of the robots tether getting stuck. A test of the new tether is to determine of it can overcome such difficulties.

In this test the tether was wrapped around two massive cylinders (Diameter of $25 \mathrm{~cm}$ ), containing $18 \mathrm{~L}$ water in a single $S$ curve. An over head view is shown in Fig. 9). This configuration exemplifies tether locking due to friction. The cylindrical shape allowed maximum contact with the hose and consequently created a sever locking case scenario. The

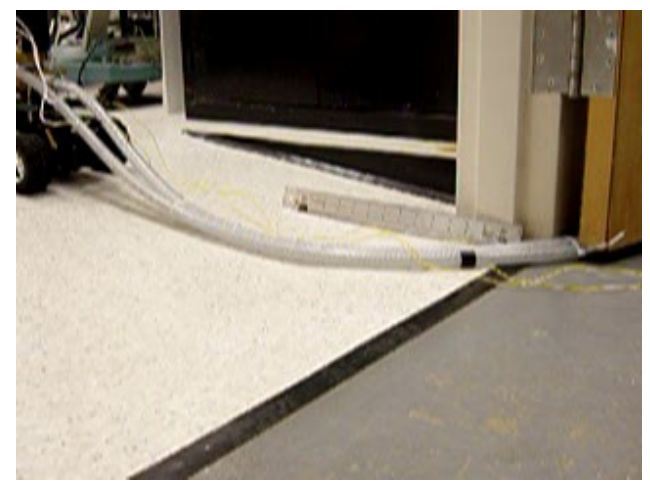

Fig. 10. Tether caught under door 

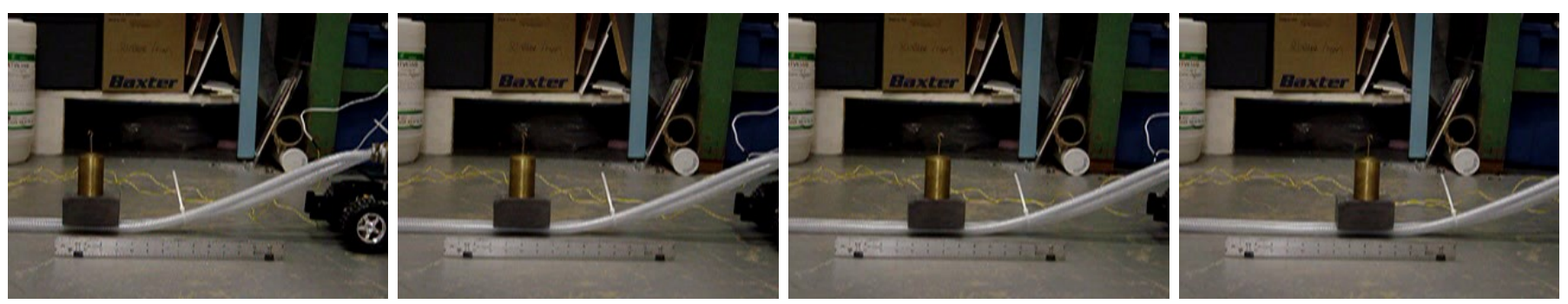

Fig. 11. Video frames from vehicle drag tests

vehicle, or a human for that matter, could not move the tether at all. When the valve was turned on and off repetitively however, the vehicle was then able to pull the tether slowly with each jerk. Another more realistic version of this test was done with the hose caught around a corner and underneath a door. The result was the same as the vehicle pulling hoses around water jugs. The vehicle could not pull forward against the locked tether but with the tether actuated the vehicle could pull forward. A video frame from this test is shown in Figure 10.

The last drag test determined the robots ability to pull the additional weight of larger tethers. With the vehicle and the tether in a line, a block of lead and a brass weight, weighing a total of $6 \mathrm{~kg}$, was placed on top of the tether providing increased drag. This is a fair test since the additional weight of the lead and brass do not contribute to the water hammer induced force but do increase drag.

Again, the vehicle could not move forward on its own. With the tether engaged the vehicle was able to pull forward with its additional burden. The vehicles progress is shown in a sequence of video frames in Fig. 11

\section{B. Skin Test}

The objective of this test was to see if the tether could move its own weight with out having a robot dragging it. To do this the valve mounted on a platform over metal ball casters, the skinned tether was pulsed repetitively. This allowed the tether to push the valve around with out relying on a dragging robot. Although slow, the skin was able to move the hose forward along its path pushing the valve forward. Given the weight of the cart and the valve, the skin on the hose performed well. While preliminarily, the biasing skin design shows promise as a technique to maximize motion due to the water hammer effect.

\section{Direct Force Measurements}

The force measured for the $0.635 \mathrm{~cm}$ diameter hose is show in Fig. 12 and the $1.27 \mathrm{~cm}$ diameter hose is show in Fig. 13. The flow rate for the $0.635 \mathrm{~cm}$ diameter hose was measured at $8.1 \times 10^{-5} \mathrm{~m}^{3} / \mathrm{s}$. Resulting in an initial velocity of $2.6 \mathrm{~m} / \mathrm{s}$ and an effective tube length of $0.836 \mathrm{~m}$. For the $1.27 \mathrm{~cm}$ diameter hose the flow rate was measured at $8.2 \times 10^{-5} \mathrm{~m}^{3} / \mathrm{s}$ and an effective tube length of $8.54 \mathrm{~m}$. The initial velocity is $0.650 \mathrm{~m} / \mathrm{s}$. The flow rates for the two hoses are approximately

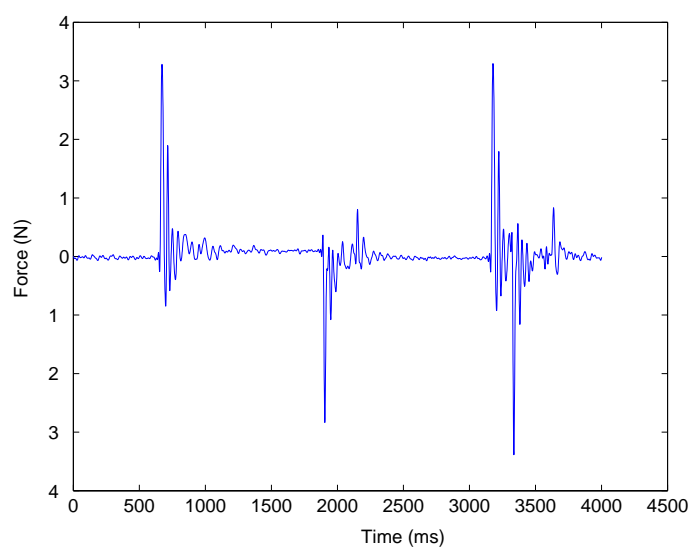

Fig. 12. Measured force with $.635 \mathrm{~cm}$ ID hose

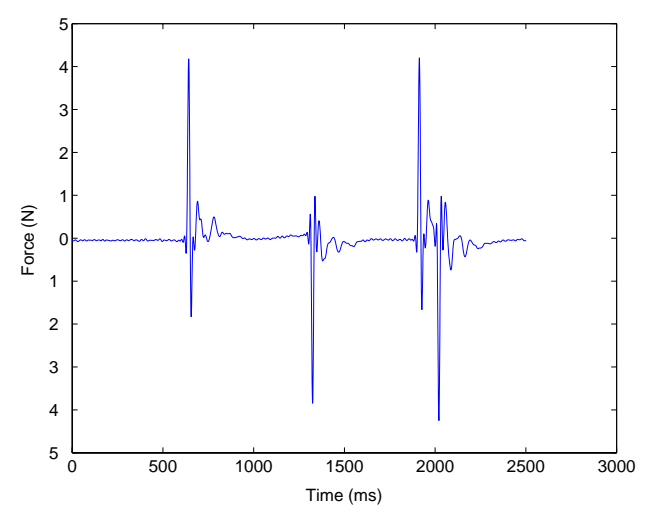

Fig. 13. Measured force with $1.27 \mathrm{~cm}$ ID hose

equivalent due to "choked flow" at the valve which has a internal diameter of only $2.38 \mathrm{~mm}$.

Despite the similar flow rates, the $0.635 \mathrm{~cm}$ hose force data is different in two ways from the force data of the $1.27 \mathrm{~cm}$. First, the peak of forces are slightly different. Second, in the larger hose, the profile of the recoil force has less ringing than the smaller hose. Both of these can be attributed to the two hoses having slightly different structural properties corresponding to different $K$ and $B$ values in the simulation. The two forces for the two hoses are shown in Fig. 14.

The motion due to water hammer will have opposite direction in the in-flow and out-flow hoses if water source and 


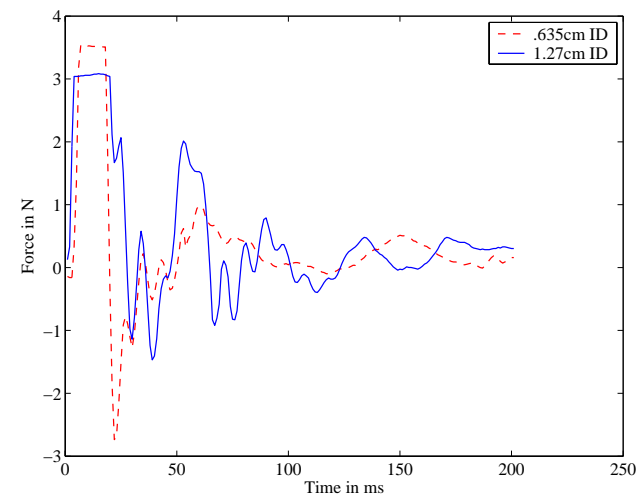

Fig. 14. Comparison of measured forces with $.635 \mathrm{~cm}$ and $1.27 \mathrm{~cm}$ ID hoses.

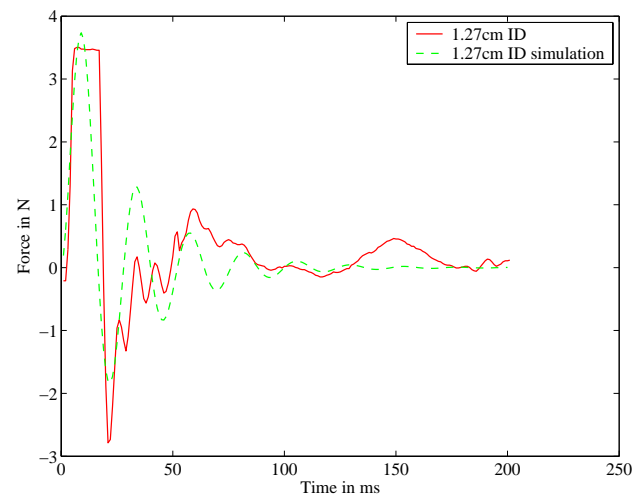

Fig. 15. Simulated and measured forces for $1.27 \mathrm{~cm}$ ID hoses simulation parameters are $\tau=1 \times 10^{-2}, M=.0633 \mathrm{~kg}, \dot{x_{0}}=.64 \mathrm{~m} / \mathrm{s}, B=5$, and $K=4000$

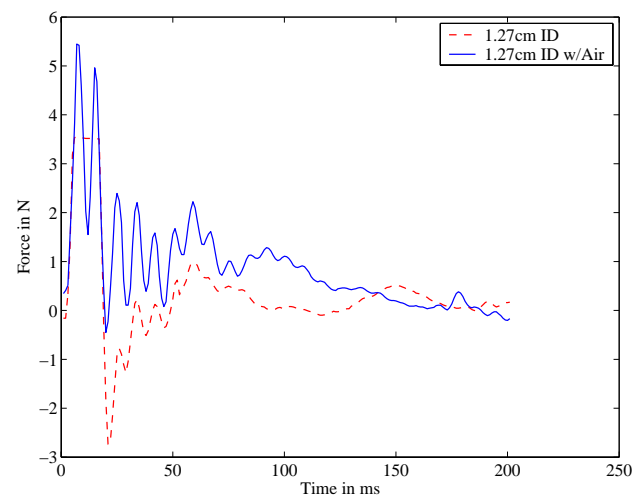

Fig. 16. Measured forces with $1.27 \mathrm{~cm}$ ID hose with and with out air bleed on out-flow side of the valve sink are at the same location. Minimizing the movement in the out-flow or returning hose is important so that the in-flow and out-flow hoses can be tied together. If both hoses are allowed to move their motions will, to some extent, cancel. The elimination of cavitation and minimization of motion in the return line is necessary to help maximize the water hammer effect. To reduce return line motion, an air bleed mechanism was added to the outflow side of the valve. This consisted of a check valve on a T-joint. Fig. 16 show the force on this modified valve. The minimization of recoil and cavitations was very noticeable. The recoil force was either eliminated or decreased compared to the non-air bleed tether. The introduction of air acutely increased the peak measured force. The out-flow line remained relatively steady while the in-flow line jerked, giving the tether its motion.

\section{DISCUSSION}

This paper presents a preliminary design for a new mobile robot tether. The experiments and drag tests have shown water hammer to be a reasonable way to actuate a tether. The pulsing tether was shown to be capable of overcoming locked conditions and moving forward with a frictionally biased skin or by being pulled by small robot. This design will increase the capabilities of rescue robots in several areas including maneuverability and range.

Although the tether advanced slowly, these demonstrations showed that the tether motion reduces the friction between high frictional surfaces. These tests also show the ability of the tether to free itself if caught. A way to prevent getting caught in the first place is to insure the tether has slack in it while traveling around a sharp corners. The frictionally biased skin could play a major role here.

This work is preliminary and as such there remains many design aspects that will merit further study. For example further investigation is needed on skin design, longer tethers, and chaining of multiply valves. We expect that gating multiple valves will present a number of interesting problems. The frictional bias of the skin could be improved by using a different material or possibly a different design all together. If a biasing skin is to be used on actual urban search and rescue (USAR) tethers it will need the ability to change direction so the tether can move backward as well as forward. In addition, since lab environments do not accurately simulate the chaos of a real collapsed building testing at a USAR simulated disaster site would be useful.

Lastly, the engineering of light wight, fast closing, compact valves and flexible, yet strong, hoses will increase the capabilities of these tethers.

\section{ACKNOWLEDGMENTS}

The authors would like to thank the Center for RobotAssisted Search and Rescue (CRASAR) for a wealth of information about search and rescue robots, tethers, and real disaster sites. We would also like thank Chris Wagner for help in deriving a simplified model for water hammer and Amy 
Kerdok for developing a way to bleed air in to the outflow side of the valve preventing cavitation.

\section{REFERENCES}

[1] J. Casper, "Human-robot interactions during the robot-assisted urban search and rescue response at the world trade center," Master's thesis, Computer Science and Engineering,University of South Florida, Apr. 2002.

[2] A. Jacoff, E. Messina, and J. Evans, "A standard test course for urban search and rescue robots," in Proc. IEEE Performance Metrics for Intelligent Systems Workshop, Gaithersburg, MD, Aug. 2000.

[3] M. R. Blackburn, H. R. Everett, and R. T. Laird, "After action report to the joint program office: Center for robotic assisted search and rescue (crasar) related efforts at the world trade center," Space and Naval Warfare Systems Command, Tech. Rep. TD 3141, Aug. 2002.

[4] L. W. Mays and Y.-K. Tung, Hydrosystems Engineering and Management. McGraw-Hill. 